\title{
Injury of the external branch of the superior laryngeal nerve in thyroid surgery
}

\author{
Andre S. Potenza, Vergilius J. F. Araujo Filho, Claudio R. Cernea \\ Department of Surgery, Head and Neck Surgery Service of the Hospital das Clínicas, Faculty of Medicine of the University of Sao Paulo, Sao Paulo, \\ Brazil \\ Contributions: (I) Conception and design: AS Potenza, CR Cernea; (II) Administrative support: All authors; (III) Provision of study materials or \\ patients: AS Potenza, CR Cernea; (IV) Collection and assembly of data: AS Potenza, CR Cernea; (V) Data analysis and interpretation: AS Potenza, \\ CR Cernea; (VI) Manuscript writing: All authors; (VII) Final approval of manuscript: All authors. \\ Correspondence to: Andre S. Potenza. 91, Av. Paulista - room 110; Sao Paulo - SP, 01311-000, Brazil. Email: apotenza@hotmail.com; Claudio R. \\ Cernea. 267, Al. Franca; Sao Paulo - SP, 01422-000, Brazil. Email: cerneamd@uol.com.br.
}

\begin{abstract}
The external branch of the superior laryngeal nerve (EBSLN) innervates the cricothyroid muscle (CTM) to promote lengthening and thinning of the vocal fold, thus increasing voice pitch. The close relation with the superior thyroid vessels (STV) puts the EBSLN in risk every time the superior pole of the thyroid is dissected. It travels downward to innervate the CTM, lateral to the thyroid cartilage and to the inferior pharyngeal constrictor muscle (IPCM), being eventually covered by this muscle fibers as it approaches its entry point. During its descending course, the EBSLN curves and crosses the STV posteriorly. The lower this crossing occurs in the neck, the higher the risk of surgical damage to the nerve by transection, traction, entrapment, thermal damage or disrupted blood supply. The chances of surgical trauma are also increased by size and weight of the specimen, shorter neck length and non-white ethnicity. Voice changes following thyroid surgery are common and multifactorial. The actual rate of vocal impairment due to EBSLN injury is unclear, since changes to the everyday speaking voice can be minimal and laryngeal findings are usually subtle and controversial. CTM electroneuromyography (EMG) is the most accurate tool to diagnose abnormal EBSLN conductivity, but it is technically difficult and barely applicable in routine practice. Recommended approaches to prevent injury include: (I) individual distal ligature of the STV by the thyroid capsule; (II) visual identification of the nerve and its trajectory and (III) electrostimulation with either observation of CTM twitch or intraoperative nerve monitoring (IONM) via dedicated endotracheal tube electrodes. There is accumulating evidence that a combination of visual and standardized electrophysiological EBSLN identification with meticulous division of the STV improves preservation rates. IONM bears the additional benefits of prognostication, quantification and documentation of neural function once it allows intraoperative laryngeal EMG.
\end{abstract}

Keywords: Intraoperative neurophysiological monitoring; laryngeal nerves; laryngeal nerve injuries; superior laryngeal nerve; thyroid gland; thyroid surgery

Submitted Jun 12, 2017. Accepted for publication Jun 19, 2017.

doi: 10.21037 /gs.2017.06.15

View this article at: http://dx.doi.org/10.21037/gs.2017.06.15

The external branch of the superior laryngeal nerve (EBSLN) is at risk of injury during thyroid operations when dissection of the superior pole and ligation of the superior thyroid vessels (STV) are carried out. The rates of injury to this nerve are highly variable in the literature, but can be as high as $58 \%$ (1). The EBSLN is the sole motor nerve to the cricothyroid muscle (CTM) and its dysfunction results in lowered voice fundamental frequency, lowered voice projection, fatigue and inability to achieve high-frequency sounds. 
Although the contribution of EBSLN to the myriad of vocal changes that may follow thyroid surgery is unclear, it is felt that singers and those who make professional use of the voice, such as lawyers, teachers and broadcasters, are more significantly affected by the subtle changes related to its injury. On the other hand, the perception of an abnormal voice impairs the quality of life and decreases the general health in many ways (2) and affected patients may be unable to shout for help, for example. From that perspective, EBSLN injury poses a threat to handicap all patients undergoing thyroid operations.

\section{Anatomy of the EBSLN}

The superior laryngeal nerve arises from the nodose ganglion of the vagus nerve. Approximately $1.5 \mathrm{~cm}$ bellow its origin and posterior to the internal carotid artery, it divides into an internal and an external branch $(3,4)$. Such bifurcation can occur at the level of the ganglion in about $5 \%$ of the individuals $(3,5)$ and the EBSLN eventually arises directly from the vagus nerve (6). The internal branch runs inferior to the greater cornu of the hyoid bone and pierces the thyrohyoid membrane to innervate the mucosa of the epiglottis, part of the anterior wall of the vallecula, the aryepiglottic folds, the interarytenoid muscles and the inferior constrictor muscle (4). The external branch crosses the common carotid artery posteriorly, crosses the middle sympathetic ganglion medially and then runs lateral to the thyroid cartilage along the pretracheal fascia superficial to the inferior pharyngeal constrictor muscle (IPCM) and travels to the CTM parallel to the superior thyroid artery (STA). Close to the lower edge of the thyroid cartilage it is usually covered by muscle fibers of the IPCM $(3,7,8)$. The nerve crosses the STA posteriorly as it approaches the superior thyroid pole. Monfared et al. (4) described that the blood supply to the EBSLN is mainly offered by the cricothyroid artery, a branch of the STA, while Yalcin et al. (9) more recently described its irrigation either from the posterior or the anterior branches of the STA, but rarely from its trunk.

The EBSLN was measured as thin as $0.2 \mathrm{~mm}$ in cadaveric studies (3) and less than $0.55 \mathrm{~mm}$ in vivo (10). It is usually encountered within the sternothyroid-laryngeal triangle, described by Moosman and DeWeese (11) in a study of 200 cadavers dissections. The boundaries of this triangle are the sternothyroid muscle superiorly, the thyroid and cricoid cartilages with the cricothyroid and pharyngeal constrictors medially, and the retracted upper thyroid lobe laterally and inferiorly (11). The avascular space between the medial aspect of the superior thyroid pole and the CTM has also been pointed out as a surgical landmark where the nerve can be steadily encountered $(12,13)$. Chuang et al. (13) dissected 86 EBSLNs from 43 Chinese adult cadavers and concluded that the inferior cornu of the thyroid cartilage also served as a reliable guide to find the external branch.

Right before entering the CTM or within it, the nerve bifurcates to innervate the pars recta and the pars obliqua of this muscle. A third muscle belly has been recently described (14). By counterstaining neural tissue with Sihler's stain, Wu et al. (15) dissected 27 human larynges and found a connection between the distal EBSLN and the recurrent laryngeal nerve (RLN) in $44 \%$ of cases, with either an intramuscular branch entering the thyroarytenoid muscle (TAM) or an anastomosis with the RLN just prior to its entrance into the TAM. Similarly, Nasri et al. (16) reported in a canine model the presence of crossinnervation to the TAM in 3 of 7 dogs, with the bulk of the activity concentrated in the anterior third of the vocal cords. The microdissection study of 90 human larynges by Sañudo et al. (17) found that a branch of the EBSLN continued to join the ventral branch of the RLN in $68 \%$ of the cadavers, as Maranillo et al. (18) found a submucosal neural connection between the EBSLN and the RLN in the paraglottic space in $85 \%$ of 103 human larynges under $\times 4$ to $\times 6$ magnification. This so-called "human communicating nerve" is of special interest in intraoperative nerve monitoring (IONM) of the EBSLN, for it allows the recording of electroneuromyography (EMG) waveforms elicited by electrical stimulation by endotracheal surface electrodes (19). This finding is reinforced by several studies that found clear-cut TAM activity provoked by EBSLN stimulation in $70 \%$ to $100 \%$ of patients undergoing thyroid surgery (20-24). Darr et al. (22) found that EBSLN-evoked potential of the TAM could be recorded in all cases when a tube with dedicated surface EMG electrodes in contact with the anterior aspect of the vocal cords was employed.

Different nomenclatures that focus on EBSLN topography and the correlated risk of injury are proposed in the literature. They suggest that the relationship among the nerve and the STA and vein is the most important surgical landmark the surgeon should perceive to prevent injury.

Cernea et al. (25) proposed their nomenclature in 1992 and since then this has been the most extensively acknowledged classification. It categorizes the position of the EBSLN in relation to the superior pole of the thyroid or the STV, as follows: 
* Type 1: the nerve crosses the superior vessels more than $1 \mathrm{~cm}$ above a plane tangent to the edge of the superior pole;

* Type 2a: it crosses the vessels within $1 \mathrm{~cm}$ above the upper limit of the superior pole

* Type 2b: the nerve curves in its travel to the CTM bellow the upper edge of the superior pole. This position represents the greatest risk of injury and has been described by the author in $14 \%$ of patients with small lobes and in 54\% of patients with very large goiters $(25,26)$.

A similar classification was later proposed by Kierner et al. (27), adding a fourth category. In Kierner's type 4 EBSLN, the nerve travels dorsally to the STA and crosses its branches immediately above the upper pole of the thyroid gland (27).

Whereas these two authors undertook cadaveric dissections, Friedman et al. (8) reported their experience in thyroid operations in a retrospective study with 884 patients and over a thousand EBSLNs examined. They offered another designation, based on the descendent course of the trunk of the EBSLN as related to the IPCM:

* Type 1 of Friedman: the EBSLN descends its entire course superficially and laterally to the IPCM until it reaches the CTM;

* Type2: the nerve deepens into the lower portion of the IPCM, being partially covered by its fibers;

* Type 3: the nerve runs its whole course covered and protected by the IPCM. This is the situation in which the nerve cannot be visually identified in the operative field. Here, electrical stimulation through IONM plays a major role in mapping out its position (8).

In 2009, Selvan et al. (10) carried out a prospective study of 35 total thyroidectomies with 70 EBSLNs being mapped through recorded compound muscle action potentials and EMG. The authors proposed a new classification founded on the STV and the cricoid cartilage as landmarks, and it is as follows:

* Selvan's type 1a EBSLN: the nerve is located within $1 \mathrm{~cm}$ of the entry of the vessels into the gland, either anterior or between its branches and within $3 \mathrm{~cm}$ from the cartilage;

* Selvan's type 1b: the nerve is posterior to the vessels, but within $1 \mathrm{~cm}$ of the entry of the STV in the gland. This is the grade that poses the greatest risk of injury in this nomenclature;

* Type 2: the nerve is located within 1 to $3 \mathrm{~cm}$ of the entry of the superior vessels into the gland or within
3 to $5 \mathrm{~cm}$ from the cricoid cartilage;

* Type 3: the nerve is between 3 and $5 \mathrm{~cm}$ of the entry of the vessels or more than $5 \mathrm{~cm}$ from the thyroid cartilage (10).

\section{Risk factors for surgical injury of the EBSLN}

Given the difficulty in diagnosing abnormal conductivity of the EBSLN, the definite incidence of injury is deemed unknown. The rates of reported surgical damage vary depending on the methods relied on to diagnose injury. When laryngoscopy and voice analysis were used, palsy rates ranged from 0 to $6 \%(7,20,28,29)$. When the more accurate laryngeal electroneuromyography (LEMG) was included in the analysis, higher rates were reported and reached $58 \%(1,19,23,24,30,31)$.

Hazard can occur by entrapment of the nerve in the ligature of the superior pole vessels, transection, stretching and thermal damage $(23,24)$. Nerve ischemia has also been considered to cause dysfunction (9) and the level of training has been implied by one of the authors (CRC) as one of the factors influencing outcomes (30). Cernea et al. (30) showed in a prospective randomized trial that there was significant difference in the rates of EBSLN paralysis accessed by LEMG between 25 operations done by a senior attendant with a $12 \%$ rate of paralysis versus 25 conducted by secondyear residents under supervision, with a $28 \%$ palsy rate $(\mathrm{P}=0.0123)$.

Regarding the surgical techniques proposed to prevent damage, three approaches are currently described:

(I) Individual ligature of the branches of the STV, as distal and as close to the thyroid capsule as possible, without the need to visually identify the nerve $(29,32)$;

(II) Attempting visual identification of the nerve before the ligature of the superior pole (12,33-35);

(III) Use of electrical neural stimulation with visualization of CTM contraction with or without IONM with endotracheal surface electrodes $(8,20$ 22,24,30,36-39).

Bellantone et al. (29) published a prospective trial including 459 upper pole ligations in 289 patients followed with phoniatric evaluation, videostrobolaryngoscopy and spectrographic analysis, in which patients were randomized to receive either thyroidectomy with visual identification of the EBSLN or with individual ligature of the superior pole vessels close to the capsule, but no attempt to search for the nerve. In neither of the groups they found vocal or 
endoscopic changes that could be accounted for EBSLN palsy. Lekacos et al. (32) also reported that no injury to the EBSLN took place when the branches of the STA were divided separately. In contrast, Droulias et al. (40) reproduced the steps of a thyroidectomy in 24 cadavers, clamped the STV adjacent to the superior thyroid pole and found out that the EBSLN was entrapped in most cases. In defense of visual identification, Hurtado-López et al. (33) published a prospective randomized blind study comparing 50 superior poles dissected without an attempt to seek for the EBSLN, with 18 poles in which the surgeons searched for it. Transcutaneous EMG of the CTM was performed after 6 months and clinically significant EBSLN injuries were less frequent in patients who had their nerves explored (8\% versus $20 \%$ ).

The aforementioned classifications stratify the EBSLN position according to the risk of injury and stand on the general belief that nerves in a lower spot in relation the superior pole are prone to increased danger. From that perspective, visual or electrophysiological identification of the nerve are felt to render a safer management of the superior pole.

In fact, by applying IONM to 400 EBSLNs at risk, Dionigi et al. (23) confirmed a significant decrease between pre-dissection and post-dissection amplitudes of evoked electrophysiological responses among nerves type $2 \mathrm{a}$ and type $2 \mathrm{~b}$ of the Cernea's nomenclature, as they remained unchanged for type 1 . Amplitudes decreased from $321 \pm 79$ to $159 \pm 57$ microvolts $(\mathrm{P}=0.04)$ for type $2 \mathrm{a}$ and from $371 \pm 38$ to $129 \pm 44$ microvolts $(\mathrm{P}=0.03)$ for $2 \mathrm{~b}$. The final amplitude fell at least $25 \%$ in $18 \%$ of type $2 \mathrm{~b}$ nerves, $11 \%$ of nerves $2 \mathrm{a}$ and only $5 \%$ of nerves type $1(\mathrm{P}=0.01)$, suggesting that dissection can be difficult and hazardous when $2 \mathrm{a}$ and $2 \mathrm{~b}$ nerves are present. Moreover, loss of EBSLN signal occurred in $2.8 \%$ of type $2 \mathrm{a}$ and $3 \%$ of $2 \mathrm{~b}$ nerves, but not in type 1 .

Significant thyroid enlargement is one of the main indications for surgery and a risk factor for injury at the same time. Examining large goiters, Cernea et al. (26) showed from 9 patients with 13 nerves at risk that the prevalence of type $2 \mathrm{~b}$ EBSLNs was as high as $54 \%$. The mean thyroid mass was $430 \mathrm{~g}$ and the length of the lobes was $10 \mathrm{~cm}$ on average in this series, suggesting that when the gland is markedly enlarged, the superior pole occupies a higher position in the neck and is more closely related to the EBSLN in its descending course (26).

In the retrospective cohort of Ravikumar et al. (41), with 912 nerves at risk, $93 \%$ of the EBSLNs were found by visual identification alone. With respect to thyroid function, Cernea's type 1 nerves were found in $23 \%$ of nontoxic goiters and only in $7 \%$ of toxic goiters $(\mathrm{P}<0.001)$. Cernea's $2 \mathrm{~b}$ nerves were present in $39 \%$ of the toxic goiters versus $5 \%$ of non-toxic $(\mathrm{P}<0.001)$. Although there was a significant association between toxicity and thyroid volume, multivariate analysis showed clear association of type $2 \mathrm{~b}$ nerves with hyperthyroidism ( $\mathrm{OR}=6.425)$ and with large goiters $(\mathrm{OR}=438.5)$. Moreover, the authors reported that no type I nerves were seen in goiters larger than $50 \mathrm{cc}$, but type $2 \mathrm{~b}$ was far more frequent among goiters above $50 \mathrm{cc}$, with a prevalence of $89 \%$ versus $6 \%$ in smaller lobes $(\mathrm{P}<0.001)$. Other authors also reported correlation between large goiters and high-risk type $2 \mathrm{~b}$ EBSLNs. Aina and Hisham (12) claimed that this subtype was present in $51 \%$ of 78 goiters heavier than $100 \mathrm{~g}$ in their 202 operated sides, and Pagedar et al. (34) saw 2 b nerves in $48 \%$ when the specimen weighted more than $50 \mathrm{~g}$.

It is unclear how ethnicity affects the topography and the risk of injury to the EBSLN. Aina and Hisham (12) found that the presence of type $2 \mathrm{~b}$ nerves in Kuala Lumpur was more prevalent in the Indian population as compared to Chinese and Malayan patients, albeit this difference was not statistically significant. Nevertheless, the prevalence of heavy goiters was lower (23\% of specimens over $100 \mathrm{~g}$ ) in Indian patients. Chuang et al. (13) also claimed that there was an increased frequency of nerves in a high-risk position $(78 \%)$ in the Chinese population. Hurtado-Lopez et al. (42), however, did not find a correlation between thyroid mass and unfavorable nerve location, but the prevalence of nerves at higher risk spots was high in this series, being reported in up to $78 \%$ of the abnormal lobes and $72 \%$ of the non-pathological contralateral lobe. The authors also suggest some association between ethnicity and increased threat. In the study of Cernea $\mathrm{et}$ al. (25), higher risk EBSLNs were significantly more common in the non-white population, also suggesting some influence of ethnicity on the surgical anatomy of the EBSLN.

Furlan et al. (43) carried out 72 cadaveric dissections and found significant differences between the three groups of the Cernea's classification regarding individual high and thyroid volume. The authors advocate that the taller the subject, the further the EBSLN is from the superior pole of the thyroid.

The proximity between the laryngeal nerves and the main thyroid vessels also raised the concern of neural injury by energy-based devices (EBD) that have been broadly employed throughout neck surgeries (44). In a prospective randomized trial with 206 thyroidectomy patients, Arslan 
et al. (45) reported that the use of the harmonic scalpel was safe and did not increase de rates of EBSLN injury when postoperative laryngostroboscopy was analyzed. The authors emphasize that the device should not be applied within less than $2 \mathrm{~mm}$ from neural structures and should not be continuously activated for longer than 15 seconds. This appears to be the only publication to target the risk of thermal lesion to the EBSLN, but several authors investigated the exposure of the RLN to energy, and we speculate that their findings are amenable to comparison with the EBSLN, but keeping in mind that the external branch is thinner and likely more vulnerable.

Yang et al. (46) studied the effects of the electrotome, the Harmonic scalpel and the Ligasure device on 24 beagles. Neither the Harmonic nor the Ligasure caused structural damage to the RLN nor any significant decrease on EMG amplitudes at 5, 3 and $1 \mathrm{~mm}$ distances, but the electrotome was hazardous at a $1-\mathrm{mm}$ distance and was associated with decreased evoked EMG amplitudes and microstructural neural damage under light and electron microscopy. In a rabbit model under evoked EMG monitoring, Jiang et al. (47) found that a harmonic scalpel at routine power levels should be at least $2 \mathrm{~mm}$ away from the RLN and should not be activated for longer than 3 seconds. In a porcine model under nerve monitoring, Wu et al. (48) found that the safe distance between the tip of the harmonic scalpel and the RLN was $1 \mathrm{~mm}$ and that it should be cooled for 10 seconds or cooled on muscle for 2 seconds prior to reuse. Lee et al. (49) reported on 9 dogs that the safe distance from the harmonic scalpel was $3 \mathrm{~mm}$, above which no vocal cord palsy and no histologic damage was noticed.

Yalcin et al. (9) hypothesized that injury to the distal blood supply to the nerve during dissection of the superior pole and its resulting ischemia also might be implied as one mechanism of neural dysfunction, especially among type $2 \mathrm{~b}$ nerves. These authors carried out 36 cadaveric microdissections with 26 dissections on the left side and 26 on the right and reported the patterns of irrigation of the EBSLN in detail. The arterial supply originated from the posterior glandular branch of the STA in $50 \%$ of sides dissected, from the anterior glandular branch in $44 \%$ and eventually from its trunk, from the infrahyoid branch and from its bifurcation. The mean arterial diameters among those distinct patterns of branching ranged from 0.56 to $0.85 \mathrm{~mm}$.

\section{EBSLN function and dysfunction}

The CTM contributes to lengthening of the vocal fold, thus affecting vocal fundamental frequency, which clinically translates into voice "pitch" (50). The cricothyroid joints allow two types of motion: rotation around the frontal axis and horizontal sliding of the cricoid cartilage in the sagittal axis leading to posterior displacement of the arytenoids with lengthening and thinning of the vocal folds (51).

The recognition of EBSLN injury is challenging. Mild changes to the speaking voice have been described, as the singing voice is more severely affected. Symptoms are described among several authors as a voice that is weak, breathy, monotonous and characterized by compression on the pitch range with inability to achieve high pitch tasks (52-55). Patients may complain of weakness, tightness, and increased effort to speak $(52,55)$. Aerodynamic measures have shown increased subglottic pressures and decreased airflow rates when the CTM is paralytic (56), as well as increased jitter (55).

Laryngeal rotation, with the posterior glottis rotated toward side of the weakness is frequently reported $(31,52,53,56,57)$. Abelson and Tucker (58) created a model of acute CTM paralysis on the larynges of 4 young volunteers under local anesthesia of the EBSLN and stated that such rotation happens dynamically during phonatory activity, mainly because the cricoid cartilage moves as the thyroid cartilage remains fixed. The authors also described that the aryepiglottic fold is shortened on the side of the paralysis (58). There may be bowing and high mismatch with inferior displacement of the vocal cord on the affected side $(31,52,54,59)$, as well as vocal fold hypomobility, mucosal asymmetrical waveform excursion and phase asymmetry $(31,54)$.

A considerable effort in the search for signs that could more accurately diagnose CTM palsy is noticeable in the literature. Rubin et al. (60) proposed that vocal fold hypomobility during fatiguing repetitive phonatory tasks could be diagnostic of EBSLN palsy. However, when mild vocal fold lag is the case, variable degrees of compensation from the unaffected muscles can make hypomobility patterns unreliable to disclose EBSLN dysfunction, as stated by Heman-Ackah and Barr (61) in a retrospective study comparing clinical scenarios, laryngeal findings and LEMG. Mendelsohn et al. (62) also reported on phase asymmetry and described a videostroboscopic pattern of motion of vocal cords "dashing against each other", as if the normal vocal cord is chasing the paralyzed one. Contrary to most of the reiterated findings above, Roy et al. (56) did not report evidences of vocal fold hypomobility, nor a consistent pattern of laryngeal rotation. In a very elucidative 
prospective study of acute injury, these authors examined 10 vocally normal men under lidocaine block of the right EBSLN with laryngeal EMG verification. Subjects were asked to perform a series of vocal tasks and the records of their flexible videolaryngoestroboscopy were analyzed by eleven blinded experts, who found deviation of the petiole of the epiglottis in $60 \%$ of subjects during an upward glissando maneuver as the only consistent endoscopic finding of EBSLN block. Later, the authors published a series of 5 clinical cases of partial and complete CTM denervation (63), again describing the finding of petiole deviation to the side of the palsy, thus ruling out the role of local diffusion of lidocaine that could have interfered with their prior results and showing that such a sign was also present in chronic palsies.

Three excellent reviews on this matter agree there are no laryngeal features that are pathognomonic or that could precisely diagnose CTM dysfunction (64-66). The clinical manifestations are currently overlooked and are subject to a variable degree of nerve impairment as well. Many variables such as coexistence with RLN dysfunction, variability in the cricothyroid joint tolerance for sliding movement (64), direct surgical injury to the CTM, compensatory action of other intrinsic laryngeal muscles and laryngeal fixation because of surgical scarring may all subsidize such a controversy. In addition, the inconstant interconnections between laryngeal nerves found in microdissection studies and variable patterns of reinnervation may play a role.

LEMG is the most useful and precise method to recognize EBSLN dysfunction $(52,64)$. Nevertheless, its applicability is limited in the routine practice because it is invasive and technically difficult. Its accuracy is highly dependent on the operator's experience and injured patients might not be willing to undergo such an invasive test knowing it is uncertain how the results would change their treatment options.

As one can expect from such variability and controversy in the perception EBSLN injury and its repercussion, there is little discussion about prognosis and dedicated treatment modalities. Voice therapy is felt to be the first treatment option for EBSLN dysfunction, mainly for its safety $(64,66)$. According to Dursun et al. $(54)$, who reported on their experience with 126 patients with suspected SLN palsies, voice therapy should focus on vocal hygiene, gentle stretching exercises, singing exercises designed to build strength and prevention of muscle tension dysphasia (54). Recovery often takes many months. Surgical interventions to the biodynamics of the larynx were proposed, such as elevation of the cricoid cartilage by attaching it to the thyrohyoid muscle and surgical approximation of the anterior thyroid and cricoid cartilage and medialization thyroplasty type IV (64,66-69). Selective CTM reinnervation using muscle-nerve-muscle neurotization with ansa hypoglossi was limitedly described, with successful outcomes in both LEMG and objective voice measures in three patients (70).

\section{Intraoperative EBSLN identification and the role of IONM}

Given the lack of optimal therapeutic alternatives for EBSLN injury, "better safe than sorry" is the rule when the superior pole of the thyroid gland is being accessed. In spite of some evidence that a peripheral ligature of the individual branches of the STV is safe $(29,32)$, many other studies suggest that some sort of identification of the nerve is advised. In a prospective randomized trial of 76 patients undergoing thyroid surgery, one of the authors (CRC) compared a group patients operated by the senior surgeon wearing $\times 2.5$ magnification loupes and with the aid of a Vari-Stim nerve stimulator, with patients operated under loupe magnification only, but without nerve stimulation (30). Cernea documented a significant rate of unilateral EBSLN injury diagnosed by CTM EMG in the second group (no lesion versus $12 \%, \mathrm{P}=0.0123$ ).

Electrical stimulation helps to map out the trajectory of both laryngeal nerves and is particularly important in Cernea's type 1 nerves and in those EBSLNs that descend deep to the IPCM fibers, that are as prevalent as $20 \%$ (8). Although the nerve is away from the main dissected area in these situations, we believe that tracking its course increases protection and is strongly recommended.

Any means of electrostimulation can help by eliciting robust CTM contraction that is easily identified in the surgical field, while IONM allows to pinpoint the nerve and to record either TAM activity or direct CTM responses. EBSLN-elicited action potentials of the TAM are felt to be mediated by the human communicating nerve described earlier and virtually all cases are amenable to this monitoring technique as long as preoperative EBSLN function is intact and proper equipment set up is implemented $(19,22)$. Also, intraoperative CTM EMG can be easily performed with subdermal EMG needle electrodes (37-39). The International Neural Monitoring Study Group (INMSG) published in 2013 its guidelines for intraoperative monitoring of the EBSLN (19). The algorithms in this 
document contribute greatly to the subject of EBSLN preservation by offering a systematic standardized approach.

Few studies describe excellent rates of unassisted visual identification of the EBSLN. In the non-randomized prospective study of Aina and Hisham (12) with 151 patients undergoing thyroid surgery, $92 \%$ of the EBSLNs were identified. Further use of a neural stimulator at $0.6 \mathrm{~mA}$ improved the identification rate in $5 \%$. Pagedar and Freeman (34) reviewed the charts of 112 patients that underwent thyroidectomies with 178 poles dissected and found an identification rate of $98.3 \%$ without neural stimulation. In the prospective case series of LochWilkinson (35), 86\% of the EBSLNs were seen in 50 total thyroidectomies and electrostimulation helped to find only 1 additional EBSLN.

This was in contrast with many other prospective studies in which rates of EBSLN visual recognition were noticeably lower in the arm without electrostimulation. Dionigi et al. (36) visually identified $42 \%$ of the EBSLNs in 72 video-assisted thyroidectomies under a $30^{\circ} 5 \mathrm{~mm}$ endoscope, while $84 \%$ were found with the aid of IONM $(\mathrm{P}<0.05)$. Similarly, Lifante et al. (37) found $21 \%$ of the nerves in the unmonitored arm of mini-incision thyroidectomies under local anesthesia versus $66 \%$ in monitored patients $(\mathrm{P}=0.03)$. Barczynski et al. (20) randomized 210 patients to undergo thyroidectomy without or with nerve monitoring and reported a rate of identification of $34 \%$ versus $84 \%$ respectively $(\mathrm{P}<0.01)$. More recently, Uludag et al. (24) documented visual identification-only in $35 \%$ of the cases in which EBSLN recognition was attempted in this study, as compared to $65 \%$ after searching it with the probe. Hurtado-López et al. (71) reported an identification rate of $97 \%$ of cases under IONM versus $79 \%$ without it.

It must be pointed out that visually identified fibers may not always be the actual nerve when CTM twitch or EMG confirmation are not explored, as other structures could be mistaken for the external branch (10). It is felt that different surgical techniques with different degrees of exposure of the sternothyroid-laryngeal triangle, such as the division of the sternothyroid muscle in Pagedar's series (34), could account for such discrepancy among identification rates $(20,24)$. On the opposite pole of surgical bed exposure, the reports on robotic (72), video-assisted (36), and mini-incision local anesthesia thyroidectomies $(37,38)$ defend that nerve monitoring is feasible and useful in providing, at least, electrophysiological identification of the EBSLN when the field is limited.
Phonation changes are the main outcomes of EBSLN injury. However, voice anomalies following thyroid surgery are somewhat common, multifactorial and may reflect not only laryngeal nerves injury, but also arytenoid trauma, cricothyroid dysfunction, strap muscle malfunction, lesion of the perithyroidal neural complex, laryngeal fixation and even psychological reaction (20). Lifante et al. (37) reported on thyroidectomies under minimal dissection and regional anesthesia, thus putting aside the possible interference of intubation and damage to the strap muscles on the postoperative voice. They documented that nerve monitoring lead to improvement in patient accessed voice-quality. In 2012, Barczynski et al. (20) published a randomized controlled trial of 210 thyroidectomies attempting to visualize the EBSLN in all cases and with further support of IONM in the study group, followed by videostrobolaryngoscopy and objective voice measures of maximum phonation time, voice level and fundamental frequency. IONM was associated with a lower rate of transient EBSLN paresis judged by endoscopy (1\% vs. 5\% without IONM, $\mathrm{P}=0.02)$ and with transiently decreased voice parameters at $2-3$ weeks after surgery, but not after 3 months.

Masuoka et al. (39) randomly assigned 252 patients with 405 EBSLNs at risk to undergo thyroid surgery with a conventional technique assisted by a Vari-Stim 3 nerve stimulator or with IONM, both set at a $1 \mathrm{~mA}$ stimulus. They concluded that IONM significantly improved not only the visual identification rate of the EBSLN, but also the stimulatory identification rate as compared to the VariStim 3 nerve stimulator. Moreover, the subjective voice impairment was lower in the IONM arm among female patients, but the voice performance measurements did not show significant differences in voice level, frequency of high pitched voice and maximum phonation time.

Dionigi et al. (23) analyzed 400 EBSLNs intraoperatively under IONM and carried out a validation of Cernea's classification. They did find that this nomenclature predicted the risk of EBSLN stress reflected by significant decreases of CTM EMG and increasing rates of loss of signal among the crescent risk-categories 1, 2a and 2b. Also, systematic neural stimulation helped to pinpoint the site of injury in 3 of 9 cases of loss of EBSLN conductivity.

In the more recent trial of Uludag et al. (24), 133 patients were randomized to undergo thyroidectomy with no attempt to visually identify the EBSLN versus with EBSLN identification through IONM. EBSLN injury was the primary endpoint measured by intraoperative CTM 
EMG with needle electrodes. Such method seems to carry the benefit of providing the actual rates of EBSLN palsy. IONM significantly prevented EBSLN damage, with injury rates of $1 \%$ in the IONM-group versus $9 \%$ when dissection of the superior pole was unassisted $(\mathrm{P}=0.01)$.

In addition to enhancing EBSLN identification, neural monitoring can be employed to preclude the presence of the nerve within the pedicles to be clamped and divided. As the INMSG recommends (19), a true negative stimulation confirmed by both the lack of electromyographical response and absent CTM twitch following probe-stimulation of the tissue to be divided should be the rule before cutting through vessels and connective tissue around the superior pole. Also, electrostimulation can be further used to map out the site of injury should transection or EBSLN entrapment occur and loss of signal subside. The plausibility to remove a clip or a tie suture that is involving the nerve is the rationale for some to prefer ligatures over EBDs when dealing with the superior pole of the thyroid gland $(19,24)$.

In contrast with routine IONM of the RLN, in which a proof that the whole nerve is intact and prognostication of neural function can be achieved by stimulation of the Vagus nerve, EBSLN's functional integrity can be registered if electrostimulation is applied proximally to the upper ligated stumps of the STV in the surgical field $(23,24)$. Dionigi et al. (23) proposed that the algorithm for IONM should include initial ( $S 1)$ and post-dissection (S2) EBSLNstimulations, as these maneuvers may serve the purpose of documentation that the nerve has been searched and preserved.

We agree that combining blunt dissection and meticulous individual division of the branches of the STVs close to the thyroid capsule with the ritual of seeing the nerve and mapping its trajectory are the best procedures to avoid hazard to the EBSLN. EBD can be used at a safe distance of several millimeters away from the nerve and the surgeon must observe the cooling time of the active tip of the instrument during repetitive activation. Special attention should be paid to cases of large goiters and nerves at high risk categories. IONM assists EBSLN preservation in many ways. It also provides quantitative data and the opportunity to perform intraoperative EMG may help to disclose the actual rates of EBSLN paralysis and paresis following thyroid surgery, thus improving future research.

\section{Footnote}

Conflicts of Interest: The authors have no conflicts of interest to declare.

\section{References}

1. Jansson S, Tisell LE, Hagne I, et al. Partial superior laryngeal nerve (SLN) lesions before and after thyroid surgery. World J Surg 1988;12:522-7.

2. Benninger MS, Ahuja AS, Gardner G, et al. Assessing outcomes for dysphonic patients. J Voice 1998;12:540-50.

3. Kambic V, Zargi M, Radsel Z. Topographic anatomy of the external branch of the superior laryngeal nerve. Its importance in head and neck surgery. J Laryngol Otol 1984;98:1121-4.

4. Monfared A, Gorti G, Kim D. Microsurgical anatomy of the laryngeal nerves as related to thyroid surgery. Laryngoscope 2002;112:386-92.

5. Furlan JC, Brandão LG, Ferraz AR, et al. Surgical anatomy of the extralaryngeal aspect of the superior laryngeal nerve. Arch Otolaryngol Head Neck Surg 2003;129:79-82.

6. Durham CF, Harrison TS. The surgical anatomy of the superior laryngeal nerve. Surg Gynecol Obstet 1964;118:38-44.

7. Lennquist S, Cahlin C, Smeds S. The superior laryngeal nerve in thyroid surgery. Surgery 1987;102:999-1008.

8. Friedman M, LoSavio P, Ibrahim H. Superior laryngeal nerve identification and preservation in thyroidectomy. Arch Otolaryngol Head Neck Surg 2002;128:296-303.

9. Yalcin B, Develi S, Tubbs RS, et al. Blood supply of the terminal part of the external branch of the superior laryngeal nerve. Surg Today 2015;45:1160-5.

10. Selvan B, Babu S, Paul MJ, et al. Mapping the compound muscle action potentials of cricothyroid muscle using electromyography in thyroid operations: a novel method to clinically type the external branch of the superior laryngeal nerve. Ann Surg 2009;250:293-300.

11. Moosman DA, DeWeese MS. The external laryngeal nerve as related to thyroidectomy. Surg Gynecol Obstet 1968;127:1011-6.

12. Aina EN, Hisham AN. External laryngeal nerve in thyroid surgery: recognition and surgical implications. ANZ J Surg 2001;71:212-4.

13. Chuang FJ, Chen JY, Shyu JF, et al. Surgical anatomy of the external branch of the superior laryngeal nerve in Chinese adults and its clinical applications. Head Neck 2010;32:53-7.

None. 
14. Mu L, Sanders I. The human cricothyroid muscle: three muscle bellies and their innervation patterns. J Voice 2009;23:21-8.

15. Wu BL, Sanders I, Mu L, et al. The human communicating nerve. An extension of the external superior laryngeal nerve that innervates the vocal cord. Arch Otolaryngol Head Neck Surg 1994;120:1321-8.

16. Nasri S, Beizai P, Ye M, et al. Cross-innervation of the thyroarytenoid muscle by a branch from the external division of the superior laryngeal nerve. Ann Otol Rhinol Laryngol 1997;106:594-8.

17. Sañudo JR, Maranillo E, León X, et al. An anatomical study of anastomoses between the laryngeal nerves. Laryngoscope 1999;109:983-7.

18. Maranillo E, León X, Quer M, et al. Is the external laryngeal nerve an exclusively motor nerve? The cricothyroid connection branch. Laryngoscope 2003;113:525-9.

19. Barczyński M, Randolph GW, Cernea CR, et al. External branch of the superior laryngeal nerve monitoring during thyroid and parathyroid surgery: International Neural Monitoring Study Group standards guideline statement. Laryngoscope 2013;123 Suppl 4:S1-14.

20. Barczynski M, Konturek A, Stopa M, et al. Randomized controlled trial of visualization versus neuromonitoring of the external branch of the superior laryngeal nerve during thyroidectomy. World J Surg 2012;36:1340-7.

21. Potenza AS, Phelan EA, Cernea CR, et al. Normative intra-operative electrophysiologic waveform analysis of superior laryngeal nerve external branch and recurrent laryngeal nerve in patients undergoing thyroid surgery. World J Surg 2013;37:2336-42.

22. Darr EA, Tufano RP, Ozdemir S, et al. Superior laryngeal nerve quantitative intraoperative monitoring is possible in all thyroid surgeries. Laryngoscope 2014;124:1035-41.

23. Dionigi G, Kim HY, Randolph GW, et al. Prospective validation study of Cernea classification for predicting EMG alterations of the external branch of the superior laryngeal nerve. Surg Today 2016;46:785-91.

24. Uludag M, Aygun N, Kartal K, et al. Contribution of intraoperative neural monitoring to preservation of the external branch of the superior laryngeal nerve: a randomized prospective clinical trial. Langenbecks Arch Surg 2016. [Epub ahead of print].

25. Cernea CR, Ferraz AR, Nishio S, et al. Surgical anatomy of the external branch of the superior laryngeal nerve. Head Neck 1992;14:380-3.

26. Cernea CR, Nishio S, Hojaij FC. Identification of the external branch of the superior laryngeal nerve (EBSLN) in large goiters. Am J Otolaryngol 1995;16:307-11.

27. Kierner AC, Aigner M, Burian M. The external branch of the superior laryngeal nerve: its topographical anatomy as related to surgery of the neck. Arch Otolaryngol Head Neck Surg 1998;124:301-3.

28. Jonas J, Bähr R. Neuromonitoring of the external branch of the superior laryngeal nerve during thyroid surgery. Am J Surg 2000;179:234-6.

29. Bellantone R, Boscherini, M, Lombardi, CP, et al. Is the identification of the external branch of the superior laryngeal nerve mandatory in thyroid operation? Results of a prospective randomized study. Surgery 2001;130:1055-9.

30. Cernea CR, Ferraz AR, Furlani J, et al. Identification of the external branch of the superior laryngeal nerve during thyroidectomy. Am J Surg 1992;164:634-9.

31. Teitelbaum BJ, Wenig BL. Superior laryngeal nerve injury from thyroid surgery. Head Neck 1995;17:36-40.

32. Lekacos NL, Miligos ND, Tzardis PJ, et al. The superior laryngeal nerve in thyroidectomy. Am Surg 1987;53:610-2.

33. Hurtado-López LM, Pacheco-Alvarez MI, MontesCastillo MDLL, et al. Importance of the intraoperative identification of the external branch of the superior laryngeal nerve during thyroidectomy: electromyographic evaluation. Thyroid 2005;15:449-54.

34. Pagedar NA, Freeman JL. Identification of the external branch of the superior laryngeal nerve during thyroidectomy. Arch Otolaryngol Head Neck Surg 2009; 135:360-2.

35. Loch-Wilkinson TJ, Stalberg PL, Sidhu SB, et al. Nerve stimulation in thyroid surgery: is it really useful? ANZ J Surg 2007;77:377-80.

36. Dionigi G, Boni L, Rovera F, et al. Neuromonitoring and video-assisted thyroidectomy: a prospective, randomized case-control evaluation. Surg Endosc 2009;23:996-1003.

37. Lifante JC, McGill J, Murry T, et al. A prospective, randomized trial of nerve monitoring of the external branch of the superior laryngeal nerve during thyroidectomy under local/regional anesthesia and IV sedation. Surgery 2009;146:1167-73.

38. Inabnet WB, Murry T, Dhiman S, et al. Neuromonitoring of the external branch of the superior laryngeal nerve during minimally invasive thyroid surgery under local anesthesia: a prospective study of 10 patients. Laryngoscope 2009;119:597-601.

39. Masuoka H, Miyauchi A, Higashiyama T, et al. Prospective randomized study on injury of the external branch of the superior laryngeal nerve during thyroidectomy comparing 
intraoperative nerve monitoring and a conventional technique. Head Neck 2015;37:1456-60.

40. Droulias C, Tzinas S, Harlaftis N, et al. The superior laryngeal nerve. Am Surg 1976;42:635-8.

41. Ravikumar K, Sadacharan D, Muthukumar S, et al. EBSLN and Factors Influencing its Identification and its Safety in Patients Undergoing Total Thyroidectomy: A Study of 456 Cases. World J Surg 2016;40:545-50.

42. Hurtado-Lopez LM, Zaldívar-Ramírez FR. Risk of injury to the external branch of the superior laryngeal nerve in thyroidectomy. Laryngoscope 2002;112:626-9.

43. Furlan JC, Cordeiro AC, Brandão LG. Study of some "intrinsic risk factors" that can enhance an iatrogenic injury of the external branch of the superior laryngeal nerve. Otolaryngol Head Neck Surg 2003;128:396-400.

44. Dionigi G, Wu CW, Kim HY, et al. Safety of energy based devices for hemostasis in thyroid surgery. Gland Surg 2016;5:490-4.

45. Arslan K, Erenoglu B, Dogru O, et al. Is the superior laryngeal nerve really safe when using harmonic focus in total thyroidectomy? A prospective randomized study. Asian J Surg 2017. [Epub ahead of print].

46. Yang X, Cao J, Yan Y, et al. Comparison of the safety of electrotome, Harmonic scalpel, and LigaSure for management of thyroid surgery. Head Neck 2017;39:1078-85.

47. Jiang H, Shen H, Jiang D, et al. Evaluating the safety of the Harmonic Scalpel around the recurrent laryngeal nerve. ANZ J Surg 2010;80:822-6.

48. Wu CW, Chai YJ, Dionigi G, et al. Recurrent laryngeal nerve safety parameters of the Harmonic Focus during thyroid surgery: Porcine model using continuous monitoring. Laryngoscope 2015;125:2838-45.

49. Lee KE, Jee HG, Kim HY, et al. Development of a canine model for recurrent laryngeal injury by harmonic scalpel. Lab Anim Res 2012;28:223-8.

50. Hirano M, Ohala J, Vennard W. The function of laryngeal muscles in regulating fundamental frequency and intensity of phonation. J Speech Hear Res 1969;12:616-28.

51. Arnold GE. Physiology and pathology of the cricothyroid muscle. Laryngoscope 1961;71:687-753.

52. Bevan K, Griffiths MV, Morgan MH. Cricothyroid muscle paralysis: its recognition and diagnosis. J Laryngol Otol 1989;103:191-5.

53. Tanaka S, Hirano M, Umeno H. Laryngeal behavior in unilateral superior laryngeal nerve paralysis. Ann Otol Rhinol Laryngol 1994;103:93-7.

54. Dursun G, Sataloff RT, Spiegel JR, et al. Superior laryngeal nerve paresis and paralysis. J Voice 1996;10:206-11.

55. Roy N, Smith ME, Dromey C, et al. Exploring the phonatory effects of external superior laryngeal nerve paralysis: An In vivo model. Laryngoscope 2009;119:816-26.

56. Roy N, Barton ME, Smith ME, et al. An in vivo model of external superior laryngeal nerve paralysis. Laryngoscope 2009;119:1017-32.

57. Adour KK, Schneider GD, Hilsinger RL. Acute superior laryngeal nerve palsy: analysis of 78 cases. Otolaryngol Head Neck Surg 1980;88:418-24.

58. Abelson TI, Tucker HM. Laryngeal findings in superior laryngeal nerve paralysis: a controversy. Otolaryngol Head Neck Surg 1981;89:463-70.

59. Tsai V, Celmer A, Berke G, et al. Videostroboscopic findings in unilateral superior laryngeal nerve paralysis and paresis. Otolaryngol Head Neck Surg 2007;136:660-2.

60. Rubin AD, Praneetvatakul V, Heman-Ackah Y, et al. Repetitive phonatory tasks for identifying vocal fold paresis. J Voice 2005;19:679-86.

61. Heman-Ackah YD, Barr A. Mild Vocal Fold Paresis: Understanding Clinical Presentation and Electromyographic Findings. J Voice 2006;20:269-81.

62. Mendelsohn AH, Sung MW, Berke GS, et al. Strobokymographic and videostroboscopic analysis of vocal fold motion in unilateral superior laryngeal nerve paralysis. Ann Otol Rhinol Laryngol 2007;116:85-91.

63. Roy N, Smith ME, Houtz DR, et al. Laryngeal features of external superior laryngeal nerve denervation: revisiting a century-old controversy. Ann Otol Rhinol Laryngol 2011;120:1-8.

64. Sulica L. The superior laryngeal nerve: function and dysfunction. Otolaryngol Clin North Am 2004;37:183-201.

65. Roy N. Denervation of the external branch of the superior laryngeal nerve: laryngeal and phonatory features. Curr Opin Otolaryngol Head Neck Surg 2011;19:182-7.

66. Orestes MI, Chhetri DK. Superior laryngeal nerve injury: effects, clinical findings, prognosis, and management options. Curr Opin Otolaryngol Head Neck Surg 2014;22:439-43.

67. Thompson JW, Ward PH, Schwartz IR. Experimental studies for correction of superior laryngeal paralysis by fusion of the thyroid to cricoid cartilages. Otolaryngol Head Neck Surg 1984;92:498-508.

68. Nasseri SS, Maragos NE. Combination thyroplasty and the "twisted larynx:" combined type IV and type I thyroplasty for superior laryngeal nerve weakness. J Voice 2000;14:104-11.

69. Shaw GY, Searl JP, Hoover LA. Diagnosis and treatment 
of unilateral cricothyroid muscle paralysis with a modified Isshiki type 4 thyroplasty. Otolaryngol Head Neck Surg 1995;113:679-88.

70. El-Kashlan HK, Carroll WR, Hogikyan ND, et al. Selective cricothyroid muscle reinnervation by musclenerve-muscle neurotization. Arch Otolaryngol Head Neck Surg 2001;127:1211-5.

71. Hurtado-López LM, Díaz-Hernández PI, Basurto-Kuba
E, et al. Efficacy of Intraoperative Neuro-Monitoring to Localize the External Branch of the Superior Laryngeal Nerve. Thyroid 2016;26:174-8.

72. Kim SJ, Lee KE, Oh BM, et al. Intraoperative neuromonitoring of the external branch of the superior laryngeal nerve during robotic thyroid surgery: a preliminary prospective study. Ann Surg Treat Res 2015;89:233-9.

Cite this article as: Potenza AS, Araujo Filho VJ, Cernea CR. Injury of the external branch of the superior laryngeal nerve in thyroid surgery. Gland Surg 2017;6(5):552-562. doi: 10.21037/ gs.2017.06.15 